\title{
Associação entre variáveis antropométricas, perfil glicêmico e lipídico em mulheres idosas
}

Association among anthropometric variables, plasma glucose and lipid profile in elderly women

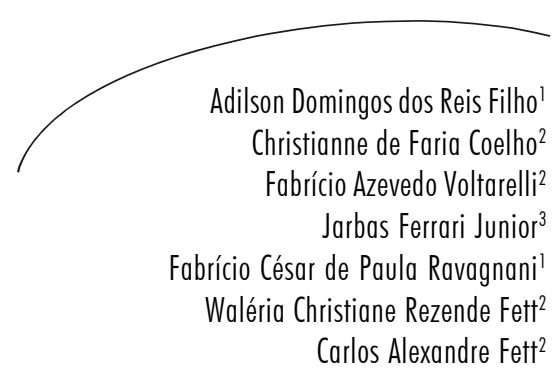

Resumo

Objetivo: Estudo transversal com o objetivo de associar variáveis antropométricas, perfil lipídico e glicêmico em 102 idosas de três centros de convivência para idosos do município de Cuiabá-MT, Brasil, com idade entre 60 e 84 anos. Métodos: Avaliaram-se índice de massa corporal, relação cintura/quadril, circunferência abdominal, percentual de gordura, glicemia em jejum, glicemia pós-prandial, hemoglobina glicada, triglicérides, colesterol total, HDL-c e LDL-c. Resultados: Houve associação significativa no grupo 60-69 anos, entre o IMC e glicemia de jejum; relação cintura-quadril e glicemia de jejum e glicemia pós-prandial; circunferência abdominal e glicemia de jejum e glicemia pós-prandial; para o grupo $>70$ anos, entre o percentual de gordura e colesterol total e a circunferência abdominal e glicemia pós-prandial. Regressão múltipla houve significância somente para o grupos > 70anos: IMC e colesterol total; relação cintura-quadril com triglicérides e com colesterol total; circunferência abdominal com glicemia de jejum, com triglicérides, com colesterol total e com LDL-c. Conclusão: As variáveis antropométricas apresentam fragilidade para associação com a bioquímica sanguínea de idosas. Entretanto, os marcadores de gordura corporal parecem estar associados à piora bioquímica, sendo a circunferência da cintura a que mais se associa especialmente em relação às mais idosas.

\section{Abstract}

Objective: Cross-sectional study aiming to associate anthropometric variables to the lipid profile and plasma glucose in 102 elderly women of three social centers for the elderly in the city of Cuiabá, Mato Grosso State, Brazil, aged between 60
Palavras-chave: Idoso. Antropometria. Glicemia. Lipoproteínas. Saúde do idoso.

\footnotetext{
Universidade de Cuiabá (UNIC), Faculdade de Educação Física. Cuiabá, MT, Brasil.

2 Departamento de Educação Física. Laboratório de Aptidão Física e Metabolismo (LAFiMe/ UFMT). Universidade Federal do Mato Grosso. Cuiabá, MT, Brasil.

3 Faculdade de Ciências Médicas. Laboratório de Aptidão Física e Metabolismo (LAFiMe/UFMT). Universidade Federal do Mato Grosso. Cuiabá, MT, Brasil.
} 
and 84 years. Methods: Were evaluated body mass index, waist/hip ratio, waist circumference, fat percentage, fasting plasma glucose, postprandial glucose, glycated hemoglobin, triglycerides, total cholesterol, HDL-c and LDL-c. Results: An association to: BMI and fasting glucose, and waist-hip ratio: fasting and postprandial glucose, and waist circumference, fasting and postprandial glycemia in the group 60-69 years. And for the group $>70$ years: percentage of total fat and cholesterol, waist circumference and postprandial glycemia. As for the multiple regression analysis indicated significant association between a BMI and total cholesterol, and waist-hip ratio, triglycerides and total cholesterol, and waist circumference, fasting glucose, triglycerides, total cholesterol and LDL-c, for all group $>70$ years. Conclusion: Among the anthropometric variables waist circumference group $>70$ years was the most sensitive to blood chemistry.
Key words: Elderly. Anthropometry. Blood Glucose. Lipoproteins. Health of the Elderly.

\section{INTRODUÇÃO}

Nos últimos anos, o envelhecimento, assim como a obesidade, vêm aumentando em vários países, sobretudo naqueles em desenvolvimento e desenvolvidos. ${ }^{1-4}$ Acompanhando esse processo de envelhecimento, modificações importantes ocorrem na composição corporal de indivíduos idosos, tais como redução da massa muscular acompanhada de aumento de tecido adiposo, principalmente aquele acumulado ao redor da cintura, ${ }^{5}$ o qual apresenta relação direta com a patogênese do diabetes mellitus tipo 2, o perfil dislipidêmico, a hipertensão arterial, o infarto agudo do miocárdio, a síndrome metabólica, entre outras doenças crônicas não-transmissíveis. ${ }^{6}$

De acordo com Weiss, ${ }^{7}$ estudos recentes têm demonstrado que o padrão de distribuição da gordura corporal é um fator determinante para o perfil metabólico e não apenas a obesidade per se. A mensuração da gordura corporal total (índice de massa corporal e percentual de gordura) e a localizada na região central (relação cintura/quadril e circunferência abdominal) vêm despertando interesse de inúmeros pesquisadores nos últimos anos, os quais buscam correlacionar o aumento do tecido adiposo com o surgimento de doenças metabólicas. Contudo, a obesidade classificada pelos valores de corte do índice de massa corporal (IMC, $\left.\mathrm{kg} / \mathrm{m}^{2}\right)^{8}$ pode não ser um fator de risco aumentado em idosos, podendo até ser considerado como protetor para indivíduos muito idosos, devido a fatores neuroendócrinos, ${ }^{9,10}$ que agem evitando efeitos catabólicos de algumas doenças.
No entanto, as técnicas consideradas padrão ouro para tal associação possuem custo elevado e alta complexidade operacional, limitando, assim, a realização de estudos populacionais. As técnicas mais simples, frequentemente utilizadas em estudos de caráter epidemiológico, ainda apresentam limitações na associação com marcadores bioquímicos de risco. ${ }^{11}$ Desta forma, o presente estudo objetivou comparar variáveis antropométricas de fácil aplicação com o perfil lipídico e glicêmico em idosas, buscando verificar quais destas poderiam melhor indicar fatores de risco associados à obesidade.

\section{METODOLOGIA}

Trata-se de um estudo transversal, descritivo e de natureza quantitativa, qualitativa e clínica, integrante do protocolo para coleta do projeto temático "Longevidade Saudável”, desenvolvido por docentes do Curso de Graduação em Educação Física e do Curso de Medicina da Universidade Federal do Mato Grosso (UFMT). O projeto temático tem o objetivo de caracterizar, descrever a prevalência, estabelecer perfis e identificar fatores de riscos entre idosos frequentadores de centros de convivência no município de Cuiabá-MT, considerando variáveis antropométricas e clínicas.

Cuiabá-MT tem população de 460.508 habitantes segundo o IBGE (2000), com 116 bairros distribuídos em quatro regionais: Oeste, Leste, Norte e Sul. São 17.329 idosos (8.221 do 
gênero masculino e 9.108 do gênero feminino), distribuídos em 120.368 domicílios da capital. Possui três centros de convivência para idosos (CCIs), mantidos pela prefeitura municipal e distribuídos nas seguintes regiões: Norte (CCI Maria Ignez França Auad, no bairro CPA III e CCI Aideê Pereira do Nascimento, no bairro Novo Horizonte) e região Leste (CCI Padre Firmo Duarte Filho, no bairro Dom Aquino). Estes CCIs oferecem oficinas de corte e costura, danças, ginástica, atendimento médico e odontológico, favorecendo assim a manutenção de um estilo de vida ativo.

Encontram-se cadastrados e frequentando os três CCIs 2.100 idosos, dos quais 1.680 são mulheres. Para seleção da amostra, foram fixados cartazes no mural informativo e realizadas palestras no período matutino e vespertino para explicar a importância das avaliações. Em seguida, foram agendados os dias para as análises, que consistiram de avaliações antropométricas e exames de sangue para identificação do perfil lipídico e glicêmico.

Como critérios de inclusão, foram observados os seguintes requisitos: ser do gênero feminino; ter idade superior a 60 anos; não ser diabética e/ ou hipertensa; não fazer uso de fármacos que alterassem a glicemia. Foram excluídas as voluntárias que apresentaram impedimentos para realização das avaliações antropométricas ou que não realizassem o exame sanguíneo.

Foram avaliadas 102 voluntárias cadastradas e frequentadoras de três centros de convivências para idosos no município de Cuiabá-MT, com idades entre 60 e 84 anos, divididas em dois grupos: $60-69$ anos (64,4 $\pm 2,5$ anos) e $>70$ anos $(73,6 \pm 3,4$ anos). Todas as voluntárias assinaram termo de consentimento livre e esclarecido aprovado pelo Comitê de Ética em Pesquisa do Hospital Universitário Júlio Müller (Protocolo $\mathrm{n}^{\circ} 330 / \mathrm{CEP}-\mathrm{HUJM} / 07$ ).

\section{Marcadores bioquímicos}

As coletas e avaliações foram de responsabilidade do Laboratório de Patologia Clínica do
Hospital do Câncer de Cuiabá-MT, Brasil. Para a obtenção da amostra sanguínea, foram observadas 12 horas de jejum, abstinência de bebidas alcoólicas no período de 72 horas e repouso de 30 minutos antes da coleta no período matutino para os seguintes marcadores bioquímicos: glicemia de jejum (GJ), hemoglobina glicada $\left(\mathrm{HbA}_{1} \mathrm{C}\right)$, triglicérides $(\mathrm{Tg})$, colesterol total (CT) e frações deste, HDLColesterol e LDL-Colesterol. A amostra de sangue para análise da glicemia pós-prandial (GPP) foi coletada após duas horas do almoço. Todas as coletas foram realizadas em triplicata, considerando a média entre elas.

As classificações atribuídas aos valores de concentrações obtidas estão de acordo com a IV Diretriz Brasileira sobre Dislipidemias ${ }^{12}$ e a American Diabetes Association, ${ }^{13}$ e estão apresentadas em suas respectivas tabelas.

Glicemia de jejum (GJ) e glicemia pós prandial (GPP): as dosagens da GJ e GPP foram realizadas por método automatizado, no aparelho CIBA CORNING 550 Express Analyzer (GMI, Inc. Ramsey: Minnesota, USA). Para análise da GJ e GPP, foram utilizados $5 \mathrm{ml}$ de soro, após ter sido centrifugado a $3.000 \mathrm{rpm}$ durante cinco minutos. O método de determinação da glicose adotado foi o enzimático colorimétrico da glicose oxidase. ${ }^{14}$

Hemoglobina glicada $\left(H b A_{1} \mathrm{C}\right)$ : para análise da $\mathrm{HbA}_{1} \mathrm{C}$, foi utilizado o sangue total colhido com anticoagulante contendo EDTA. Foi utilizado para determinação da $\mathrm{HbA}_{1} \mathrm{C}$ o método Trivelli modificado. ${ }^{15}$

Triglicérides (Tg), colesterol total (CT) e frações (HDL-c e LDL-c): para o CT, Tg e HDLc foram aplicados o método colorimétrico enzimático utilizando-se $5 \mathrm{ml}$ de soro após ter sido centrifugado a $3.000 \mathrm{rpm}$ durante cinco minutos. ${ }^{16,14} \mathrm{~A}$ fração de colesterol LDL-c foi obtida utilizando a fórmula preconizada por Friedewald et al. ${ }^{17}$ As dosagens foram realizadas com o método automatizado no aparelho CIBA CORNING 550 Express Analyzer (GMI, Inc. Ramsey: Minnesota, USA). 


\section{Composição corporal}

Para determinação do peso corporal, as voluntárias foram posicionadas em pé, no centro da plataforma da balança, com os pés unidos e braços ao longo do corpo, ${ }^{18}$ utilizando-se balança digital SOEHNLE ${ }^{\circledR}$ Professional 7755(Brasil), com capacidade para $200 \mathrm{~kg}$ e precisão de $100 \mathrm{~g}$. A estatura foi mensurada com as voluntárias descalças, em posição ereta, com os pés unidos e próximos a escala, medidas pelo estadiômetro disponível na mesma balança, com precisão de 0,5 $\mathrm{cm}$, segundo o procedimento previamente descrito por Fett et al. ${ }^{18}$

Foram mensuradas, com fita antropométrica modelo SN-4010 da marca Sanny ${ }^{\oplus}$, as circunferências de braço, antebraço, punho, cintura, abdominal, quadril, coxa e panturrilha, todas de acordo com o protocolo proposto por Pollock e Wilmore. ${ }^{19} \mathrm{O}$ cálculo da relação cintura/ quadril (RCQ) foi realizado pela divisão do perímetro da cintura $(\mathrm{cm})$ pelo perímetro do quadril $(\mathrm{cm})$. As dobras cutâneas foram aferidas por adipômetro $L A N G E^{\circledR}$ (Beta Technology Inc. Santa Cruそ: CA, US A), com pressão constante de $10 \mathrm{~g} /$ $\mathrm{mm}^{2}$ na superfície de contato e precisão de $1 \mathrm{~mm}$. As dobras cutâneas mensuradas foram: triceptal, suprailíaca e coxa, conforme o disposto em Pollock e Wilmore. ${ }^{19}$ As coletas das medidas foram realizadas em triplicata e por um único e experiente avaliador, utilizando-se a média das mesmas para o cálculo da densidade corporal, de acordo com Jackson et al. ${ }^{20} \mathrm{e}$, posteriormente, o cálculo do percentual de gordura segundo protocolo descrito por Siri. ${ }^{21}$

\section{Análise estatística}

A normalidade da amostra foi calculada pelo teste Kolmogorov-Smirnov. ${ }^{22}$ Para a determinação das correlações entre as variáveis, utilizaram-se o teste de correlação linear de Pearson para os dados paramétricos e a correlação de Spearman para dados não-paramétricos. ${ }^{22}$ Posteriormente, foi aplicado o teste de regressão linear múltipla para análise da importância relativa das diferentes variáveis. ${ }^{22} \mathrm{O}$ nível de significância foi preestabelecido em $5 \%(p<0,05)$.
O tamanho amostral foi calculado segundo a equação:

$$
\mathrm{n}=\frac{\mathrm{Z}^{2} \mathrm{P}(1-\mathrm{P}) \mathrm{N}}{\mathrm{Z}^{2} \mathrm{P}(1-\mathrm{P}) \mathrm{N}+\mathrm{E}^{2} \mathrm{P}(\mathrm{N}-1)}
$$

Onde: Z é o valor de uma distribuição normal e apresenta confiabilidade de 90\% (1,644854); P é a probabilidade da escolha de uma idosa do centro de convivência $=0,5 ;$ E é nível de erro aceitável para obtenção da amostra, neste caso, 0,05; e Né o total de idosas do centro de convivência $=1.680$.

\section{RESULTADOS}

De um total de 234 idosas inicialmente selecionadas nos três centros de convivências, apenas 110 voluntárias compareceram para a realização dos exames bioquímicos e antropométricos; destas, somente 102 (6,1\% do total de idosas dos CCIs) foram consideradas elegíveis para integrarem a pesquisa, as demais $(\mathrm{n}=8)$ foram excluídas por serem diabéticas e/ou fazerem uso de algum medicamento.

Os resultados apresentados na (tabela 1), para a faixa etária de 60-69 anos, demonstram índice de sobrepeso em relação ao IMC $\left(28,2 \pm 5,2 \mathrm{~kg} / \mathrm{m}^{2}\right)$, de obesidade para o percentual de gordura $(35,8 \pm 7,3)$, alto risco para a relação cintura-quadril $(0,86 \pm 0,06)$ e risco muito aumentado para a circunferência abdominal $(98,3 \pm 10,4)$. A glicemia em jejum e glicemia pós-prandial foram consideradas dentro da normalidade, os triglicérides e colesterol total apresentaram índices ótimos, o LDL-c $(105,1 \pm 31,3)$ manteve-se dentro dos limites desejáveis e o HDL-c e a hemoglobina glicada com risco baixo. Para a faixa etária com idade acima de 70 anos, pode-se observar IMC condizente com sobrepeso $(28,1 \pm 4,9)$, obesidade em relação ao percentual de gordura $(36,7 \pm 6,8)$, alto risco para a relação cintura-quadril $(0,85 \pm 0,09)$, risco muito aumentado para a circunferência abdominal $(98,8 \pm 10,6)$ e em relação a glicemia em jejum e glicemia pós-prandial níveis dentro da normalidade, triglicérides e colesterol total níveis ótimos, LDL-c em ótimo estado e HDL-c e hemoglobina glicada com baixo risco (tabela 1). 
Tabela 1 - Características gerais de idosas de Centros de Convivência de Cuiabá-MT, 2009.

\begin{tabular}{lccccc}
\hline \multicolumn{1}{c}{ Variáveis } & $60-69$ anos $(\mathrm{n}=61)$ & \multicolumn{2}{c}{$>$ anos $(\mathrm{n}=41)$} & \\
\hline & média & DP & média & DP & p-valor \\
Idade (anos) & 64.4 & 2.5 & 73.6 & 3.4 & $<0.0001^{*}$ \\
Estatura (m) & 1.54 & 0.05 & 1.51 & 0.05 & $0.004^{\dagger}$ \\
Massa corporal & 67.1 & 12.4 & 64.2 & 10.8 & $\mathrm{~ns}$ \\
Índice de Massa Corporal (kg/m²) & 28.2 & 5.2 & 28.1 & 4.9 & $\mathrm{~ns}$ \\
Percentual de Gordura (\%) & 35.8 & 7.3 & 36.7 & 6.8 & $\mathrm{~ns}$ \\
Massa gorda (kg) & 24.7 & 9.4 & 24.2 & 8.1 & $\mathrm{~ns}$ \\
Massa magra (kg) & 42.3 & 4.9 & 39.9 & 3.9 & $0.01^{*}$ \\
Relação Cintura/Quadril & 0.86 & 0.06 & 0.85 & 0.09 & $\mathrm{~ns}$ \\
Circunferência Abdominal (cm) & 98.3 & 10.4 & 98.8 & 10.6 & $\mathrm{~ns}$ \\
Glicemia de Jejum (mg/dL) & 81.8 & 15.0 & 85.1 & 22.3 & $\mathrm{~ns}$ \\
Glicemia Pós-Prandial (mg/dL) & 99.2 & 20.5 & 106.5 & 39.8 & $\mathrm{~ns}$ \\
Triglicérides (mg/dL) & 129.1 & 61.5 & 126.6 & 59.8 & $\mathrm{~ns}$ \\
Colesterol Total (mg/dL) & 192.1 & 30.3 & 184.6 & 37.8 & $\mathrm{~ns}$ \\
HDL-c (mg/dL) & 61.4 & 2.9 & 61.7 & 16.0 & $0.002^{*}$ \\
LDL-c (mg/dL) & 105.1 & 31.3 & 98.6 & 36.9 & $\mathrm{~ns}$ \\
Hemoglobina Glicada (\%) & 6.0 & 0.8 & 6.2 & 1.2 & $\mathrm{~ns}$ \\
\hline
\end{tabular}

(HDL-c) Lipoproteína de alta densidade-colesterol; (LDL-c) Lipoproteína de baixa densidade-colesterol. *Teste Mann-Whitney; TTeste $t$ de Student. Nível de significância $\mathrm{p}<0.05$.

$\mathrm{Na}$ tabela 2, observa-se maior prevalência de idosas com o peso acima da normalidade para a faixa etária compreendida entre 60 e 69 anos, quando analisados IMC, sobrepeso $(\mathrm{n}=15$; $24,6 \%)$ e obesidade ( $n=16 ; 26,2 \%)$ e no grupo de idosas acima de 70 anos, sobrepeso $(n=5 ; 12,2 \%)$ e obesidade ( $\mathrm{n}=14 ; 34,2 \%)$. Na mesma tabela observa-se, também, um número excessivo de idosas apresentando valores elevados para a relação cintura-quadril, percentual de gordura e circunferência abdominal, para as duas faixas etárias. 
Tabela 2 - Distribuição das idosas segundo a faixa etária e variáveis antropométricas, Cuiabá-MT, 2009.

\begin{tabular}{|c|c|c|c|c|}
\hline & $\begin{array}{c}60-69 \\
\text { anos } \\
(\mathrm{n}=61)\end{array}$ & Prevalência \% & $\begin{array}{l}>70 \\
\text { anos } \\
(\mathrm{n}=41)\end{array}$ & Prevalência \% \\
\hline \multicolumn{5}{|l|}{$\mathrm{IMC}\left(\mathrm{kg} / \mathrm{m}^{2}\right)$} \\
\hline baixo-peso $<23$ & 8 & 13.2 & 6 & 14.6 \\
\hline normal 23 a 27,99 & 22 & 36.0 & 16 & 39.0 \\
\hline sobrepeso 28 a 29,99 & 15 & 24.6 & 5 & 12.2 \\
\hline obesidade $>30$ & 16 & 26.2 & 14 & 34.2 \\
\hline \multicolumn{5}{|l|}{ RELAÇÃO CINTURA/QUADRIL } \\
\hline baixo $<0,76$ & 2 & 3.3 & 3 & 7.3 \\
\hline moderado 0,76 a 0,83 & 19 & 31.2 & 3 & 7.3 \\
\hline alto 0,84 a 0,90 & 23 & 37.7 & 24 & 58.6 \\
\hline muito alto $>0,90$ & 17 & 27.8 & 11 & 26.8 \\
\hline \multicolumn{5}{|l|}{$\%$ GORDURA } \\
\hline excelente 16 a $20 \%$ & 3 & 4.9 & 1 & 2.4 \\
\hline bom 22 a $26 \%$ & 2 & 3.2 & 1 & 2.4 \\
\hline abaixo da média 27 a $29 \%$ & 8 & 13.2 & 6 & 14.7 \\
\hline média 30 a $31 \%$ & 7 & 11.4 & 8 & 19.6 \\
\hline acima da média 32 a $34 \%$ & 12 & 19.6 & 4 & 9.8 \\
\hline ruim 35 a $37 \%$ & 11 & 18.1 & 2 & 4.8 \\
\hline muito ruim 38 a $41 \%$ & 18 & 29.6 & 19 & 46.3 \\
\hline \multicolumn{5}{|l|}{$\begin{array}{l}\text { CIRCUNFERÊNCIA ABDOMINAL } \\
(\mathrm{cm})\end{array}$} \\
\hline$<80$ & 1 & 1.7 & 0 & 0 \\
\hline aumentado $\geqslant 80$ & 8 & 13.1 & 8 & 19.5 \\
\hline muito aumentado $\geqslant 88$ & 52 & 85.2 & 33 & 80.5 \\
\hline
\end{tabular}

IMC segundo a Organização Pan-Americana da Saúde (OPAS, 2002); \% Gordura segundo Pollock e Wilmore (1993).

Em relação aos marcadores bioquímicos sanguíneos (tabela 3), nota-se que, mesmo com os valores antropométricos elevados, não foram observadas alterações nas concentrações de glicemias de jejum e pós-prandial, triglicérides, colesterol total, HDL-c, LDL-c e hemoglobina glicada, sendo que tais valores apresentaram-se, de forma prevalente, dentro da normalidade. 
Tabela 3 - Distribuição das idosas segundo a faixa etária, perfil lipídico e glicêmico, Cuiabá-MT, 2009.

\begin{tabular}{|c|c|c|c|c|}
\hline & $\begin{array}{c}60-69 \\
\text { anos } \\
(\mathrm{n}=61)\end{array}$ & Prevalência \% & $\begin{array}{c}>70 \\
\text { anos } \\
(\mathrm{n}=41)\end{array}$ & Prevalência \% \\
\hline \multicolumn{5}{|l|}{ GLICEMIA JEJUM } \\
\hline Normal 70 a 99 mg/dL & 57 & 93.4 & 35 & 85.3 \\
\hline Intolerância 100 a 125 mg/dL & 2 & 3.3 & 5 & 12.3 \\
\hline $\begin{array}{l}\text { Diabetes } \geqslant 126 \mathrm{mg} / \mathrm{dL} \\
\text { GLICEMIA PÓS-PRANDIAL }\end{array}$ & 2 & 3.3 & 1 & 2.4 \\
\hline Normal $<140 \mathrm{mg} / \mathrm{dL}$ & 59 & 96.7 & 37 & 90.3 \\
\hline Intolerância 140 a 199 mg/dL & 2 & 3.3 & 3 & 7.3 \\
\hline \multicolumn{5}{|l|}{ TRIGLICÉRIDES } \\
\hline Ótimo $<150$ mg/dL & 46 & 75.4 & 31 & 75.6 \\
\hline Limítrofe 150 a $200 \mathrm{mg} / \mathrm{dL}$ & 8 & 13.1 & 4 & 9.8 \\
\hline Alto risco 201 a $499 \mathrm{mg} / \mathrm{dL}$ & 7 & 11.5 & 6 & 14.6 \\
\hline \multicolumn{5}{|l|}{ COLESTEROL TOTAL } \\
\hline Ótimo $<200 \mathrm{mg} / \mathrm{dL}$ & 40 & 65.6 & 32 & 78.0 \\
\hline Limítrofe 200 a 239 mg/dL & 15 & 24.6 & 4 & 9.7 \\
\hline $\begin{array}{l}\text { Alto } \geqslant 240 \mathrm{mg} / \mathrm{dL} \\
\text { HDL-colesterol }\end{array}$ & 6 & 9.8 & 5 & 12.3 \\
\hline Alto risco $<40 \mathrm{mg} / \mathrm{dL}$ & 0 & 0 & 0 & 0 \\
\hline Entre 50 e $59 \mathrm{mg} / \mathrm{dL}$ & 15 & 24.6 & 19 & 46.3 \\
\hline \multicolumn{2}{|l|}{ LDL-colesterol } & 75.4 & 22 & 53.7 \\
\hline Ótimo $<100 \mathrm{mg} / \mathrm{dL}$ & 27 & 44.3 & 20 & 48.8 \\
\hline Desejável 100 a 129 mg/dL & 21 & 34.4 & 14 & 34.1 \\
\hline Limítrofe 130 a 159 mg/dL & 9 & 14.7 & 3 & 7.3 \\
\hline Alto 160 a $189 \mathrm{mg} / \mathrm{dL}$ & 4 & 6.6 & 4 & 9.8 \\
\hline Muito alto $\geqslant 190 \mathrm{mg} / \mathrm{dL}$ & 0 & 0 & 0 & 0 \\
\hline \multicolumn{5}{|l|}{ HEMOGLOBINA GLICADA } \\
\hline$<7 \%$ & 57 & 93.4 & 38 & 92.7 \\
\hline$>7 \%$ & 4 & 6.6 & 3 & 7.3 \\
\hline
\end{tabular}

Classificações de acordo com a IV Diretriz Brasileira sobre Dislipidemias, (2007) e American Diabetes Association, (2007).

Quando correlacionados com marcadores bioquímicos sanguíneos, os valores das medidas antropométricas apresentaram pouca associação e, mesmo naqueles com significância estatística, o coeficiente de correlação mostrou-se baixo (tabela 4). Em relação aos resultados da análise de regressão múltipla, apenas o grupo de idosas acima de 70 anos obteve significância estatística entre as variáveis dependentes (glicemiadejejum, glicemia pós-prandial, triglicérides, colesterol total, LDL-c e hemoglobina glicada) e explicativas (IMC, relação cintura-quadril, circunferência abdominal eidade), segundo a tabela 5. 
Tabela 4 - Correlação entre variáveis antropométricas, perfil lipídico e glicêmico em idosas frequentadoras de centros de convivência de Cuiabá-MT, 2009.

\begin{tabular}{|c|c|c|c|c|c|c|c|c|}
\hline \multicolumn{9}{|c|}{ IDOSAS 60-69 ANOS } \\
\hline & \multicolumn{2}{|c|}{$\operatorname{IMC}\left(\mathrm{kg} / \mathrm{m}^{2}\right)$} & \multicolumn{2}{|c|}{$\% G$} & \multicolumn{2}{|c|}{ RCQ } & \multicolumn{2}{|c|}{$\mathrm{CAb}(\mathrm{cm})$} \\
\hline GJ & $\mathrm{rs}=0.26$ & $p=0.03$ & $\mathrm{rs}=0.12$ & ns & $\mathrm{rs}=0.30$ & $p=0.01$ & $\mathrm{rs}=0.35$ & $p=0.005$ \\
\hline GPP & $\mathrm{rs}=0.24$ & $p=0.05$ & $\mathrm{rs}=0.18$ & ns & $r s=0.25$ & $p=0.04$ & $\mathrm{rs}=0.31$ & $p=0.01$ \\
\hline $\mathrm{Tg}$ & $\mathrm{rs}=0.18$ & ns & $\mathrm{rs}=0.13$ & ns & $\mathrm{rs}=0.25$ & $\mathrm{p}=0.05$ & $\mathrm{rs}=0.21$ & ns \\
\hline CT & $\mathrm{rs}=0.01$ & ns & $r=0.12$ & ns & $r=-0.05$ & ns & $r=-0.02$ & ns \\
\hline HDL-c & $r s=-0.01$ & ns & $r=-0.12$ & ns & $r=-0.19$ & ns & $r=-0.07$ & ns \\
\hline LDL-c & $\mathrm{rs}=-0.05$ & ns & $r=0.11$ & ns & $r=-0.16$ & ns & $r=-0.07$ & ns \\
\hline $\mathrm{HbA}_{1} \mathrm{C}$ & $\mathrm{rs}=0.07$ & ns & $r=0.008$ & ns & $r=0.07$ & ns & $r=0.08$ & ns \\
\hline \multicolumn{9}{|c|}{ IDOSAS > 70 ANOS } \\
\hline & \multicolumn{2}{|c|}{ IMC $\left(\mathrm{kg} / \mathrm{m}^{2}\right)$} & \multicolumn{2}{|c|}{$\% \mathrm{G}$} & \multicolumn{2}{|c|}{ RCQ } & \multicolumn{2}{|c|}{$\mathrm{CAb}(\mathrm{cm})$} \\
\hline GJ & $\mathrm{rs}=0.12$ & ns & $\mathrm{rs}=0.06$ & ns & $\mathrm{rs}=0.29$ & $p=0.05$ & $\mathrm{rs}=0.29$ & $p=0.05$ \\
\hline GPP & $\mathrm{rs}=0.25$ & ns & $\mathrm{rs}=0.16$ & ns & $\mathrm{rs}=0.22$ & ns & $\mathrm{rs}=0.30$ & $p=0.04$ \\
\hline $\mathrm{Tg}$ & $\mathrm{rs}=0.20$ & ns & $\mathrm{rs}=0.24$ & ns & $\mathrm{rs}=0.13$ & ns & $\mathrm{rs}=0.26$ & ns \\
\hline CT & $r=0.17$ & ns & $r=0.37$ & $p=0.01$ & $\mathrm{rs}=0.13$ & ns & $r=0.22$ & ns \\
\hline HDL-c & $\mathrm{rs}=0.07$ & ns & $\mathrm{rs}=0.02$ & ns & $\mathrm{rs}=0.15$ & ns & $\mathrm{rs}=0.01$ & ns \\
\hline LDL-c & $r=0.08$ & ns & $r=0.26$ & ns & $\mathrm{rs}=0.03$ & ns & $r=0.09$ & ns \\
\hline $\mathrm{HbA}_{1} \mathrm{C}$ & $\mathrm{rs}=0.15$ & ns & $\mathrm{rs}=0.11$ & ns & $\mathrm{rs}=0.15$ & ns & $\mathrm{rs}=0.29$ & ns \\
\hline
\end{tabular}

(GJ) Glicemia de jejum; (GPP) Glicemia pós-prandial; (Tg) Triglicérides; (CT) Colesterol total; (HbA C) Hemoglobina glicada; (HDLc) Lipoproteína de alta densidade-colesterol; (LDL-c) Lipoproteína de baixa densidade-colesterol; (IMC) Índice de massa corporal; $(\% \mathrm{G})$ Percentual de gordura; (RCQ) Relação cintura/quadril e (CAb) Circunferência abdominal. Correlação de Spearman (rs); Correlação linear de Pearson (r); Não significante (ns). Nível de significância p $<0,05$. 
Tabela 5 - Coeficiente de regressão linear múltipla $(\beta)$ das variáveis antropométricas, idade, perfil lipídico e glicêmico em idosas frequentadoras de centros de convivência de Cuiabá-MT, 2009.

\begin{tabular}{|c|c|c|c|c|c|c|c|}
\hline & \multicolumn{7}{|c|}{ IDOSAS 60-69 ANOS } \\
\hline & GJ & GPP & $\mathrm{Tg}$ & $\mathrm{CT}$ & HDL-c & LDL-c & $\mathrm{HbA}_{1} \mathrm{C}$ \\
\hline IMC & $\beta=2.3$ & $\beta=5.6$ & $\beta=5.3$ & $\beta=0.2$ & $\beta=-0.2$ & $\beta=0.2$ & $\beta=-0.01$ \\
\hline$\left(\mathrm{kg} / \mathrm{m}^{2}\right)$ & ns & ns & ns & ns & ns & ns & ns \\
\hline$\% G$ & $\beta=-0.4$ & $\beta=1.7$ & $\beta=6.7$ & $\beta=0.3$ & $\beta=0.05$ & $\beta=-0.9$ & $\beta=-0.002$ \\
\hline & ns & ns & ns & ns & ns & ns & ns \\
\hline RCQ & $\begin{array}{c}\beta=96.2 \\
\text { ns }\end{array}$ & $\begin{array}{c}\beta=158.1 \\
n s\end{array}$ & $\begin{array}{c}\beta=454.3 \\
\text { ns }\end{array}$ & $\begin{array}{c}\beta=-84.5 \\
\text { ns }\end{array}$ & $\begin{array}{c}\beta=-16.2 \\
\mathrm{~ns}\end{array}$ & $\begin{array}{c}\beta=-155.0 \\
n s\end{array}$ & $\begin{array}{c}\beta=1.6 \\
\text { ns }\end{array}$ \\
\hline $\mathrm{CAb}(\mathrm{cm})$ & $\begin{array}{c}\beta=0.5 \\
\text { ns }\end{array}$ & $\begin{array}{c}\beta=1.1 \\
\mathrm{~ns}\end{array}$ & $\begin{array}{c}\beta=-0.6 \\
\text { ns }\end{array}$ & $\begin{array}{c}\beta=-1.2 \\
\mathrm{~ns}\end{array}$ & $\begin{array}{c}\beta=-0.01 \\
\text { ns }\end{array}$ & $\begin{array}{c}\beta=-1.0 \\
\mathrm{~ns}\end{array}$ & $\begin{array}{c}\beta=0.03 \\
\text { ns }\end{array}$ \\
\hline IDADE & $\begin{array}{c}\beta=0.02 \\
\mathrm{~ns}\end{array}$ & $\begin{array}{c}\beta=-1.0 \\
\text { ns }\end{array}$ & $\begin{array}{c}\beta=0.9 \\
\text { ns }\end{array}$ & $\begin{array}{c}\beta=-0.6 \\
\mathrm{~ns}\end{array}$ & $\begin{array}{c}\beta=-0.07 \\
\text { ns }\end{array}$ & $\begin{array}{c}\beta=-0.9 \\
\text { ns }\end{array}$ & $\begin{array}{c}\beta=-\begin{array}{c}-0.009 \\
n s\end{array} \\
\text { ns }\end{array}$ \\
\hline \multirow[t]{3}{*}{ PESO } & $\begin{array}{c}\beta=-0.6 \\
n s\end{array}$ & $\begin{array}{c}\beta=0.9 \\
\mathrm{~ns}\end{array}$ & $\begin{array}{c}\beta=26.2 \\
\text { ns }\end{array}$ & $\begin{array}{c}\beta=-11.8 \\
n s\end{array}$ & $\begin{array}{c}\beta=-2.0 \\
n s\end{array}$ & $\begin{array}{c}\beta=-18.3 \\
n s\end{array}$ & $\begin{array}{c}\beta=-0.5 \\
\text { ns }\end{array}$ \\
\hline & \multicolumn{7}{|c|}{ IDOSAS > 70 ANOS } \\
\hline & GJ & GPP & $\mathrm{Tg}$ & CT & HDL-c & LDL-c & $\mathrm{HbA}_{1} \mathrm{C}$ \\
\hline IMC & $\beta=2.5$ & $\beta=4.3$ & $\beta=14.1$ & $\beta=9.0$ & $\beta=-0.8$ & $\beta=8.4$ & $\beta=0.1$ \\
\hline$\left(\mathrm{kg} / \mathrm{m}^{2}\right)$ & ns & ns & $p=0.05$ & $p=0.03$ & ns & ns & ns \\
\hline$\% \mathrm{G}$ & $\begin{array}{c}\beta=-5.1 \\
n s\end{array}$ & $\begin{array}{c}\beta=-5.5 \\
\text { ns }\end{array}$ & $\begin{array}{c}\beta=-2.5 \\
\text { ns }\end{array}$ & $\begin{array}{c}\beta=2.2 \\
\mathrm{~ns}\end{array}$ & $\begin{array}{c}\beta=0.2 \\
\mathrm{~ns}\end{array}$ & $\begin{array}{c}\beta=6.4 \\
\text { ns }\end{array}$ & $\begin{array}{c}\beta=-0.1 \\
n s\end{array}$ \\
\hline RCQ & $\begin{array}{c}\beta=174.8 \\
\mathrm{~ns}\end{array}$ & $\begin{array}{c}\beta=153.5 \\
\text { ns }\end{array}$ & $\begin{array}{l}\beta=801.2 \\
p=0.003\end{array}$ & $\begin{array}{l}\beta=443.8 \\
p=0.004\end{array}$ & $\begin{array}{c}\beta=9.0 \\
\text { ns }\end{array}$ & $\begin{array}{c}\beta=287.6 \\
n s\end{array}$ & $\begin{array}{c}\beta=8.8 \\
\mathrm{~ns}\end{array}$ \\
\hline $\mathrm{CAb}(\mathrm{cm})$ & $\begin{array}{l}\beta=1.8 \\
p=0.02\end{array}$ & $\begin{array}{c}\beta=2.7 \\
\mathrm{~ns}\end{array}$ & $\begin{array}{c}\beta=5.4 \\
p=0.006\end{array}$ & $\begin{array}{c}\beta=3.6 \\
p=0.001\end{array}$ & $\begin{array}{c}\beta=-0.6 \\
\mathrm{~ns}\end{array}$ & $\begin{array}{l}\beta=2.7 \\
p=0.02\end{array}$ & $\begin{array}{l}\beta=0.09 \\
p=0.05\end{array}$ \\
\hline IDADE & $\begin{array}{c}\beta=-1.8 \\
\text { ns }\end{array}$ & $\begin{array}{l}\beta=-4.2 \\
p=0.04\end{array}$ & $\begin{array}{l}\beta=-6.8 \\
p=0.01\end{array}$ & $\begin{array}{l}\beta=-3.2 \\
p=0.03\end{array}$ & $\begin{array}{c}\beta=0.1 \\
\mathrm{~ns}\end{array}$ & $\begin{array}{c}\beta=-1.7 \\
n s\end{array}$ & $\begin{array}{c}\beta=-0.08 \\
\text { ns }\end{array}$ \\
\hline PESO & $\begin{array}{c}\beta=6.1 \\
\text { ns }\end{array}$ & $\begin{array}{c}\beta=25.8 \\
\text { ns }\end{array}$ & $\begin{array}{c}\beta=47.8 \\
\text { ns }\end{array}$ & $\begin{array}{c}\beta=50.5 \\
\mathrm{~ns}\end{array}$ & $\begin{array}{c}\beta=0.2 \\
n s\end{array}$ & $\begin{array}{c}\beta=47.2 \\
\text { ns }\end{array}$ & $\begin{array}{c}\beta=0.4 \\
n s\end{array}$ \\
\hline
\end{tabular}

(GJ) Glicemia de jejum; (GPP) Glicemia pós-prandial; (Tg) Triglicérides; (CT) Colesterol total; (HDL-c) Lipoproteína de alta densidade-colesterol; (LDL-c) Lipoproteína de baixa densidade-colesterol; $\left(\mathrm{HbA}_{1} \mathrm{C}\right)$ Hemoglobina glicada; (IMC) Índice de massa corporal; $(\% \mathrm{G})$ Percentual de gordura; (RCQ) Relação cintura/quadril e (CAb) Circunferência abdominal. Coeficiente de Regressão Linear Múltiplar ( $\beta$ ); Não significante (ns). Nível de significância $\mathrm{p}<0,05$.

\section{DISCUSSÃO}

No presente estudo, foram observadas prevalências aumentadas de variáveis como (relação cintura/quadril, percentual de gordura e circunferência abdominal) para ambos os grupos, sendo estas variáveis diretamente associadas às doenças crônicas não transmissíveis. ${ }^{23-26}$ Corroborando com estes achados, o estudo conduzido por Sanches-Viveros et al, ${ }^{26}$ na população mexicana indicou a associação significativa entre variáveis antropométricas aumentadas e doenças crônicas, tais quais, o diabetes mellitus tipo 2 e a hipertensão arterial.
Os resultados obtidos referentes às variáveis IMC, relação cintura-quadril, percentual de gordura e circunferência abdominal mostraramse semelhantes aos observados em outras pesquisas, ${ }^{27,28}$ que não identificaram modificações importantes entre as duas faixas etárias. No entanto, no estudo realizado por Koster et al., ${ }^{29}$ os indivíduos com IMC dentro da normalidade, mas com a circunferência abdominal aumentada, apresentavam maior risco de mortalidade. Dessa forma, a mensuração da circunferência abdominal surge como uma prática viável no sentido de complementar a medida de IMC, visto que nem sempre o IMC aumentado é sinônimo de alguma 
doença crônica não-transmissível. De acordo com Weiss et al., ${ }^{30}$ indivíduos idosos se beneficiariam do IMC elevado devido ao estoque de energia em forma de gordura auxiliar na proteção contra efeitos catabólicos.

Evidências clínicas sugerem que a coexistência de diabetes e obesidade central está fortemente associada à gordura geral, ${ }^{11}$ mas no presente estudo não foi diagnosticado nenhum caso de diabetes em ambos os grupos, mesmo nas idosas com a RCQ e a CAb aumentadas. Outras evidências sugerem que o padrão de distribuição central do tecido adiposo apresenta correlação com diabetes mellitus e com as doenças cardiovasculares, independentemente da obesidade global. ${ }^{28}$ Contudo, na presente pesquisa, os dados referentes à obesidade global (IMC e \%G) e à obesidade central (RCQ e CAb) não confirmaram tal associação.

A obesidade central tem sido associada com diminuição da tolerância à insulina, alterações na homeostase glicose/insulina, redução na remoção de insulina e decréscimo na utilização de glicose estimulada pela insulina. ${ }^{11}$ Resultado semelhante ao disposto anteriormente foi relatado por Tabata et al., ${ }^{31}$ que encontraram forte associação entre o aumento da CAb e resistência à insulina. Porém, em nosso estudo, mesmo com as voluntárias apresentando valores elevados de CAb, não foram encontradas alterações na GJ, tampouco na GPP, divergindo, assim, do disposto anteriormente por Vazquez ${ }^{11}$ e Tabata et al. ${ }^{31}$ No estudo desenvolvido por Shen et al., ${ }^{32}$ foram encontradas melhores associações da CAb com indicadores de síndrome metabólica se comparada ao \%G. Esse fato foi confirmado em nossa amostra, principalmente para as idosas acima de 70 anos.

Em estudo realizado por Alhamdan, ${ }^{33}$ foram encontradas associações positivas entre o conteúdo de triglicérides e o IMC, a relação cintura-quadril e circunferência abdominal; e associações negativas entre o HDL-c e as variáveis antropométricas indicadoras de gordura central, quais sejam: relação cintura-quadril e circunferência da cintura. No presente estudo, foram encontradas associações entre o IMC e glicemia de jejum e glicemia pós-prandial; relação cintura-quadril e glicemia de jejum, glicemia pós-prandial e triglicérides; circunferência abdominal e glicemia de jejum e glicemia pós-prandial, todos para o grupo de idosas com idade entre 60-69 anos. Já as idosas com mais de 70 anos apresentaram associações entre o percentual de gordura e colesterol total; relação cintura-quadril e glicemia de jejum; circunferência abdominal e: glicemia de jejum e glicemia pós-prandial. Desta forma, sugere-se que a circunferência abdominal seria um indicador de risco metabólico mais confiável para a população idosa.

Para reforçar este raciocínio, foi aplicado o teste de regressão linear múltipla no intuito de encontrar um indicador que pudesse explicar melhor tais suspeitas; com isso, pode-se detectar que somente as variáveis relação cintura-quadril e circunferência abdominal do grupo de idosas acima de 70 anos apresentaram significância na regressão múltipla, sendo a variável circunferência abdominal a que mais importância teve na explicação dos desfechos, corroborando assim Zahorska-Markiewicz, ${ }^{34}$ que afirma ser a circunferência abdominal o melhor preditor para o possível desenvolvimento de doenças metabólicas, se comparado ao IMC e à relação cintura-quadril.

O presente trabalho apresenta como uma de suas limitações o desenho de estudo do tipo transversal, o que impossibilita tecer conclusões a respeito da causalidade dos resultados aqui apresentados e discutidos. Outro fator limitante foi o $n$ amostral de 6,1\%; sendo assim, não foi possível extrapolar os achados desta pesquisa para a população total de idosas que frequenta os três centros de convivência. Destacamos ainda o fato de as idosas serem fisicamente ativas e talvez por isso não apresentarem alterações no perfil lipídico e glicêmico, mesmo com algumas variáveis antropométricas aumentadas.

\section{CONCLUSÃO}

Foram observadas diferenças entre os indicadores antropométricos e a associação com 
o perfil lipídico e glicêmico entre as diferentes faixas etárias. Sugere-se assim, que existe um comportamento diferenciado quanto ao aumento da massa corpórea e alterações da glicemia e lípides plasmáticos em idosas e muito idosas. Isso talvez seja um fator protetor no que se refere à perda de massa corporal que ocorre com a velhice, confundindo a relação com os marcadores

\section{REFERÊNCIAS}

1. JAM, Garcia RA. O envelhecimento da população brasileira: um enfoque demográfico. Cad Saúde Pública. 2003; 19(3): 725-33.

2. Velazquez-Alva MC, Irigoyen ME, Zepeda M, et al. Anthropometric measurements of a sixtyyear and older mexican urban group. The Journal of Nutrition, Health \& Aging 2004; 8(5): 350-354.

3. Jensen GL. Obesity among older persons: screening for risk of adverse outcomes. The Journal of Nutrition, Health \& Aging. 2006; 10(6): 510-522.

4. Cunha AS, Sichieri R. Trends in underweight, overweight and body mass index among elderly population of Brazil. The Journal of Nutrition, Health \& Aging 2007; 11(1): 15-19.

5. Perissinotto E, Pisent C, Sergi G, Grigoletto F, Enzi G. Anthropometric measurements in the elderly: age and gender differences. British Journal of Nutrition 2002; 87: 177-186.

6. Lechleitner M. Obesity and the Metabolic Syndrome in the Elderly: a Mini-Review. Gerontol 2008; 54: 253-259.

7. Weiss R. Fat distribution and storage: how much, where, and how? European J. Endocrinol 2007; 157: S39-S45.

8. WHO. Physical status: the use and interpretation of anthropometry. Geneva: World Health Organization; 1999; p.1-36

9. Weiss A, Beloosesky Y, Boaz M, et al. Body mass index is inversely related to mortality in elderly subjects. J Gen Intern Med 2007; 23(1):19-24.

10. Nicoletti I, Cicoira M, Morando G, et al. Impact of body mass index on short-term outcome after acute myocardial infarction: does excess body weight have a paradoxical protective role? Int J Cardiol 2006; 107(3): 395-399.

11. Vazquez G, Duval S, Jacobs Jr, et al. Comparison of body mass index, waist circumference, and waist/hip ratio in predicting bioquímicos. Embora frágil, a relação cinturaquadril e principalmente a circunferência abdominal foram as que mais apresentaram associação com os marcadores bioquímicos. Tal achado sugere que a relação cintura-quadril e a circunferência abdominal são variáveis importantes na avaliação de comportamento de risco metabólico para idosas. incident diabetes: a meta-analysis. Epidemiol Rev. 2007; 29: 115-128.

12. Departamento de Aterosclerose da Sociedade Brasileira de Cardiologia. IV Diretriz Brasileira Sobre Dislipidemias e Prevenção da Aterosclerose. Arq Bras Cardiol 2007; 88(1): 1-19.

13. American Diabetes Association. Diagnosis and Classification of Diabetes Mellitus. Diabetes Care 2007; 30(1): S4-S41.

14. Bergmeyer HU. Methods of Enzymatic Analysis. 2nd. Ed. New York: Academic Press; 1985.p.1205-1214.

15. Trivelli LA, Ranney HM, Lai HT. Hemoglobin components in patients with diabetes mellitus. N. Engl. J. Med 1971; 284:353-57

16. Fredrickson DS, Levy RI, Lee RS. Fat transport in lipoproteins: an integrated approach to mechanisms and disorders. New Engl. J. Med.1967; 276:34-42.

17. Friedwald WT, Levy RI, Fredrickson DS. Estimation of the Concentration of Low-Density Lipoprotein Cholesterol in Plasma, Without Use of the Preparative Ultracentrifuge. Clin. Chem. 1972; 18:499-502.

18. Fett CA, Fett WCR, Padovan GJ, et al. Mudanças no estilo de vida e fatores de risco para doenças crônicas não transmissíveis e sistema imune de mulheres sedentárias. Rev. Nutr. 2009; 22(2): 245-255.

19. Pollock ML, Wilmore JH. Exercícios na saúde e na doença: avaliação e prescrição para prevenção e reabilitação. 2.ed. Rio de Janeiro: MEDSI;1993.

20. Jackson AS, Pollock ML, Ward A. Generalized equations for predicting body density of women. Med Sci Sports Exerc. 1980; 12: 75-82.

21. Siri SE. Body composition from fluid spaces and density: analysis of methods. In: Brozek J, Henschel A eds. Techniques for Measuring Body Composition. Washington: National Research Council;1961; p.223. 
22. Dawson B, Trapp RG. Basic \& Clinical Biostatistics. ${ }^{\text {nd }}$.ed. USA: Lange Medical Books/McGraw-Hill; 1994; p.82-97.

23. Organização Pan-Americana. Informe preliminar da 36 ${ }^{\mathrm{a}}$ Reunión del Comité Asesor de Investigaciones em Salud : Encuesta Multicéntrica - Salud Bienestar y Envejecimento (SABE) em América Latina y el Caribe; 2002. [ Acesso em 15 jun 2002].Disponível em: http: // www.opas.org/program/sabe.

24. Keller HH, Østbye T. Body mass index (BMI), BMI change and mortality in communitydwelling seniors without dementia. The Journal of Nutrition, Health \& Aging. 2005; 9(5): 316-320.

25. Mairs JB. The Diabetes Prevention Program Research Group: relationship of body size and shape to the development of diabetes in the diabetes prevention program. Obesity 2006; 14(11): 2107-2117.

26. Sanchez-Viveros S, Barquera S, Medina-Solis CE, et al. Association between diabetes mellitus and hypertension with anthropometric indicators in older adults: results of the mexican health survey. The Journal of Nutrition, Health \& Aging 2008; 12(5): 327-333.

27. Elia M. Obesity in the elderly. Obes Res 2001; 9: 244S-248S.
28. Cabrera MAS, Jacob Filho W. Obesidade em idosos: prevalência, distribuição e associação com hábitos e co-morbidades. Arq Bras Endocrinol Metab. 2001; 45(5): 494-501.

29. Koster A, Leitzmann MF, Schatzkin A, et al. Waist circumference and mortality. Am J of Epidemiol 2008; 167(12): 1465-1475.

30. Weiss A, Beloosesky Y, Boaz M, et al. Body mass index is inversely related to mortality in elderly subjects. J Gen Intern Med 23(1):19-24, 2007.

31. Tabata S, Yoshimitsu S, Hamachi T, et al. Waist circumference and insulin resistance: a crosssectional study of Japanese men. BMC Endocrine Disorders 2009; 9(1): 1472-6823.

32. Shen W, Punyanitya $M$, Chen J, et al. Waist circumference correlates with metabolic syndrome indicators better than percentage fat. Obesity. 2006; 14: 727-736.

33. Alhamdan AA. Body mass índex, waist, waist to hip ratio and lipid profile in elderly subjects living in a nursing home. J. Med. Sci. 2008; 8(2): 177-181.

34. Zahorska-Markiewicz B. Metabolic effects associated with adipose tissue distribution. Advances in Medical Sciences 2006; 51: 111-14. 